\title{
Les études ichtyologiques liées à la construction du barrage de Petit-Saut : un premier bilan et des recommandations
}

\author{
Ichthyological studies relating to the construction \\ of Petit-Saut dam: preliminary assessment \\ and recommendations
}

\author{
Bernard de Mérona(1), Luis Tito de Morais ${ }^{(2)}$ \\ (1) Laboratoire d'Hydrobiologie, Centre ORSTOM de Cayenne, BP 165, Cayenne cedex, France. \\ (2) ORSTOM, BP 1434, Bouake, République de Côte d'lvoire.
}

Résumé. - La construction de barrages hydroélectriques s'accompagne le plus souvent d'études environnementales visant à évaluer et tenter de minimiser les conséquences sur l'environnement de ce type d'ouvrage. Idéalement ces études devraient intervenir tout au long du processus, depuis la phase de faisabilité jusqu'à une éventuelle stabilisation écologique des milieux. A Petit-Saut, les premières études ichtyologiques, menées avant le début de la construction, se sont d'emblée focalisées sur le site. Elles n'étaient donc pas concernées par le choix du site. Les études avant mise en eau ont permis une bonne connaissance de la systématique de l'ichtyofaune et de sa répartition spatiale dans le bassin. Elles ont apporté également quelques connaissances parcellaires de la biologie des principales espèces, et surtout permis l'initiation de deux approches nouvelles dans ce type de travail : l'écologie des juvéniles et l'étude des stratégies vitales des populations. Après mise en eau ces deux approches ont été approfondies pour suivre et tenter d'interpréter les modifications induites par le barrage. Au total, le bilan des études ichtyologiques sur le Sinnamary barré s'avère très positif. Cependant certains aspects des études environnementales pourraient être améliorés et nous formulons quelques recommandations.

Mots clés. - Amérique du Sud, Guyane, barrage, écologie, poissons.

Abstract. - Large hydroelectric projects generally include environmental studies whose purpose is to understand and try to minimize the negative impacts of river damming on the environment. Ideally, these studies should be conducted throughout the entire process of dam construction, from the feasability studies until the possible ecological stabilization 
of the ecosystems created. At Petit-Saut, the first ichthyological studies, conducted before the beginning of construction, did not contribute to decision making concerning site choice. Studies prior to the filling of the reservoir produced comprehensive data on the knowledge of the systematics and the spatial distribution of the ichtyofauna. They also produced preliminary data on the biology of the main species and, more importantly, enabled the development of two original approaches to this kind of problem: ecology of juveniles and study of life-history strategies of populations. After closure of the dam, these two approaches were broadened so as to contribute to monitoring and the interpretation of changes in fish assemblages. On the whole, the overall contribution of the ichthyological studies on the damed Sinnamary river is extremely valuable. However, some aspects of the environmental studies could be improved and certain recommendations are formulated regarding this aspect.

Key Words. - South America, French Guiana, dam, ecology, fish.

\section{INTRODUCTION}

La mise en place de barrages hydroélectriques qui vise l'utilisation d'une source d'énergie renouvelable constitue une agression majeure à l'environnement et particulièrement aux écosystèmes aquatiques. Le barrage sectionne le cours d'eau en trois tronçons écologiquement bien distincts, il modifie les conditions hydrologiques à l'aval, il transforme l'habitat lotique en habitat lentique au niveau de la retenue et il isole la partie amont de la rivière. Face à ces bouleversements, la préoccupation environnementale a le plus souvent été présente dans les réalisations de barrages (Scope, 1972).

La réalisation d'un barrage peut être décomposée en quatre phases (Scope, 1972; Ackermann et al., 1973) (fig. 1) : 1) les études de faisabilité qui conduisent à la décision de construire en un lieu donné, 2) la construction elle-même, 3) le remplissage de la retenue et la stabilisation physique, et enfin 4) l'évolution écologique du système.

Dans ce schéma il apparaît que les études d'écologie devraient débuter dès le début de la phase de faisabilité afin que les considérations environnementales soient prises en compte dans l'ensemble du processus de décision.

Dans la phase de construction il s'agit de prévoir les conséquences environnementales de l'ouvrage dans le site choisi en vue d'intégrer dans la construction d'éventuels aménagements structurels permettant de limiter les impacts négatifs.

La phase de mise en eau est essentiellement une période de surveillance. Les phénomènes physiques et chimiques $y$ sont très rapides et les conséquences sur les communautés biologiques peuvent être dramatiques. II faut donc être en mesure de détecter rapidement d'éventuelles catastrophes afin de corriger les problèmes en influant sur les opérations de mise en eau et, en particulier, 


\section{PHASES DE L'HISTOIRE DU LAC}

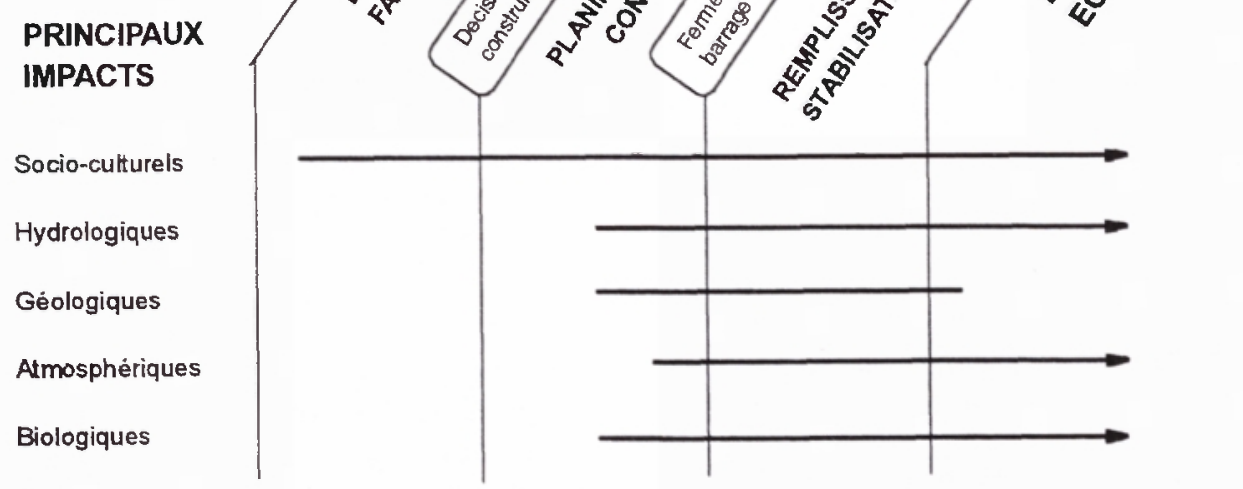

\section{ETUDES SOUHAITABLES}

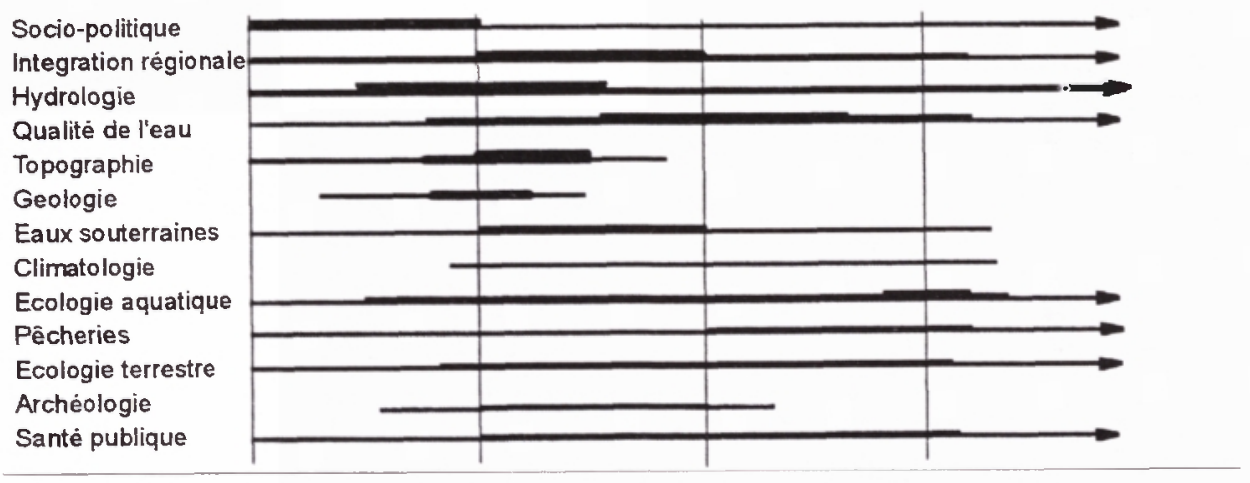

Fig. 1. - Représentation schématique des différentes phases dans l'établissement d'un barrage hydroélectrique. Modifié de SCOPE (1972).

Fig. 1. - Schematic representation of the different stages of development in a hydroelectric plan. Modified from SCOPE (1972).

l'établissement de paliers. II est également du plus grand intérêt de vérifier les hypothèses émises lors de la phase précédente.
Enfin c'est à l'issue de la phase d'évolution écologique qu'il est possible de faire le bilan des effets du barrage sur l'environnement. La pos- 
sibilité de généraliser les conséquences observées à d'autres situations dépend de plus de la compréhension des mécanismes ayant conduit à une situation de relatif équilibre de l'écosystème transformé.

Dans l'ensemble des études d'environnement, celles concernant les poissons sont fondamentales pour tout ce qui a trait aux milieux aquatiques. D'une part il s'agit souvent d'une ressource exploitée par les populations riveraines et le maintien de la qualité et de la quantité de poissons est une préoccupation_sociale et économique de premier plan (Mérona, 1985). D'autre part, les poissons ont, dans le milieu aquatique, une position trophique élevée et sont ainsi des intégrateurs de l'ensemble des phénomènes chimiques et biologiques intervenant dans leur environnement (Verneaux, 1981; Karr et al., 1986).

Le barrage hydroélectrique de $\mathrm{Pe}$ tit-Saut sur le fleuve Sinnamary en Guyane française a été fermé le 4 janvier 1994. Il s'agit d'un ouvrage de moyenne importance qui, avec une trentaine de mètres de chute, a une puissance installée de $160 \mathrm{MW}$. La fermeture a entraîné la mise en eau d'une retenue de quelques $350 \mathrm{~km}^{2}$ dans une zone de forêts denses.

Les premiers travaux d'ichtyologie menés par I'INRA dans le cadre de la construction du barrage de PetitSaut ont couvert la période 1981-1984 (Planquette et al., 1985). Ces travaux, destinés à être inclus dans la demande de concession se sont focalisés sur le bassin du Sinnamary. Des études complémentaires ont été effec- tuées entre 1989 et 1993 par l'INRA et l'ORSTOM (Tito de Morais et Planquette, 1991; Lauzanne et al., 1993; Lauzanne et al., 1995). Les études ichtyologiques se sont poursuivies après le début de la mise en eau en janvier 1994, pendant toute la phase de remplissage et jusqu'au moment présent (Lauzanne et al., 1995; Mérona et al., 1996; Mérona, 1997a).

Nous nous proposons dans ce travail de faire un bilan des études menées sur les poissons dans le cadre de l'implantation du barrage de PetitSaut sur le Sinnamary. Nous discuterons ce bilan et proposerons quelques recommandations visant à une plus grande efficacité dans la programmation des études d'environnement liées aux constructions de barrages.

\section{LES ÉTUDES ICHTYOLOGIQUES À PETIT-SAUT}

\subsection{Les acquis avant la ferme- ture du barrage}

\subsubsection{L'inventaire}

Préalable indispensable à toute analyse de l'évolution de l'ichtyofaune dans une situation donnée, l'inventaire taxonomique des poissons est une tâche particulièrement longue et délicate dans les milieux aquatiques néotropicaux dont la faune est très diversifiée et relativement mal connue.

Les premiers inventaires fournissent un chiffre de 126 espèces comprenant 109 espèces dulçaquicoles, c'est à dire celles qui fréquentent exclusivement les eaux douces, 
et 17 espèces euryhalines qui peuvent effectuer une partie de leur cycle dans les eaux saumâtres (Planquette et al., 1985). Après complément de collecte et synonymies le dernier point, réalisé en 1995, fait état d'un total de 136 espèces de ces deux catégories. II faut ajouter 21 taxons marins qui remontent à un degré ou à un autre la partie basse du cours (Lauzanne et al., 1995).

Les déterminations se sont appuyées exclusivement sur des critères morphologiques et méristiques comme c'est classiquement le cas. Si la plupart des doutes peuvent être levés par ce moyen il subsiste néanmoins quelques problèmes qui de- manderaient des méthodes plus élaborées.

Cet inventaire permet de considérer le Sinnamary comme une rivière riche en espèces si on le compare à d'autres cours d'eau d'Amérique du Sud (fig. 2), mais cette conclusion est discutable car la richesse spécifique observée est bien entendu fonction de l'intensité de l'échantillonnage. Dans le Sinnamary l'échantillonnage a été particulièrement intensif pendant plusieurs années avec des engins variés (chalut, filets maillants, explosifs, poison, pièges, lignes) et couvrant l'ensemble du cours depuis l'estuaire jusqu'au très haut cours (Saut Maïpouri). En particulier, nous avons une

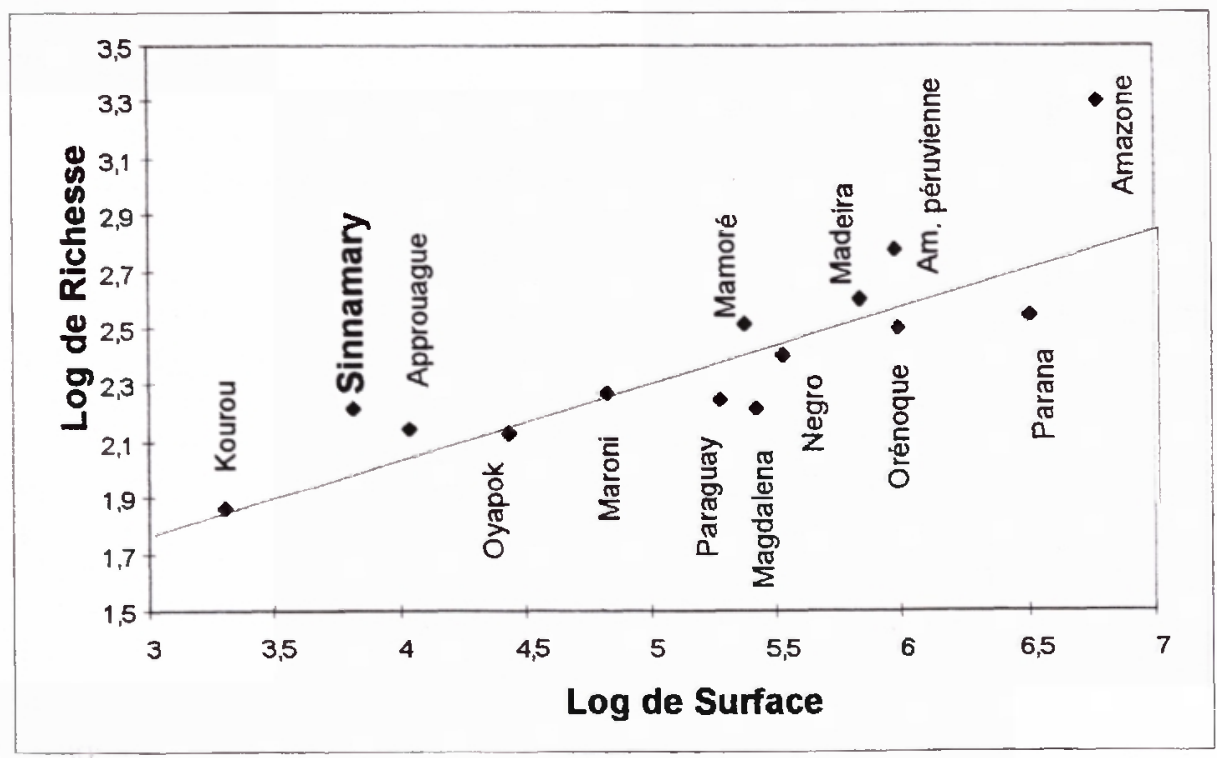

Fig. 2. - Relation entre la taille des bassins et la richesse spécifique des poissons pour quelques fleuves et rivière d'Amérique du Sud. Modifié de Lauzanne et al. (1993).

Fig. 2. - Relationship between basin area and fish specific richness in some South American Rivers. Modified from Lauzanne and al. (1975). 
bonne connaissance de la richesse en petites espèces, celles dont la taille adulte ne dépasse pas quelques $\mathrm{cm}$. C'est rarement le cas dans les inventaires réalisés dans d'autres rivières du continent.

Cet inventaire a également permis d'affirmer avec une quasi certitude qu'il n'y avait pas d'espèce endémique dans le Sinnamary et qu'ainsi, la présence du barrage ne risquait pas d'entraîner directement d'extinction d'espèces.

\subsubsection{Répartition des peuplements}

Une première étude de la répartition des espèces dans le cours du Sinnamary basée sur des échantillons limités à la moitié inférieure du cours a proposé un schéma à trois zones: la partie haute, la partie basse et la zone estuarienne (Boujard et RojasBeltran, 1988). Par la suite, l'extension du programme de collectes aux zones les plus amont du bassin et la multiplication du nombre d'échantillons a permis d'obtenir une image plus fine de la répartition des peuplements de poissons (Lauzanne et al., 1995) (fig. 3). II s'avère d'une manière générale que la zonation est peu marquée. La distribution des espèces le long du cours permet de définir trois zones en plus de la zone estuarienne. L'une appelée zone basse est située en aval de saut Vata, rapide qui constitue de fait le premier obstacle important à la remontée de poissons. L'autre appelée zone moyenne s'étend entre saut Vata et saut Takari-Tanté, autre barrière importante. Enfin la zone haute comprend l'ensemble du cours à l'amont de saut Takari-Tanté qui se trouve être la limite supérieure du barrage.

On observe que la zone moyenne qui est celle qui a disparu par la création du lac de retenue de Petit-Saut est aussi celle qui est le moins caractéristique du cours d'eau initial. Les échantillons récoltés sont proches soit de ceux de la zone amont soit de ceux de la zone aval.

\subsubsection{Eléments de biologie des espèces}

Dans ce domaine aussi les connaissances se sont accumulées au cours des études successives mais sont restées très fragmentaires à l'issue de cette phase.

Dès les premières études les grandes lignes des régimes alimentaires d'une trentaine d'espèces ont pu être déterminées par des analyses de contenus stomacaux (Planquette et al., 1985). Ces observations ont ensuite été complétées pour les poissons des criques et des sauts (Horeau et al., 1996). Parallèlement un suivi de la condition des individus a été effectué.

Fig. 3. - Carte du bassin du Sinnamary avec le profil en long et la zonation des peuplements de poissons. $A$ = selon Boujard et Rojas-Beltran (1988). B = selon Lauzanne et al. (1995).

Fig. 3. - Map of the Sinnamary basin with the longitudinal profile and the fish zonation. A : according to Boujard \& Rojas-Beltran (1988). B : According to Lauzanne et al. (1975). 


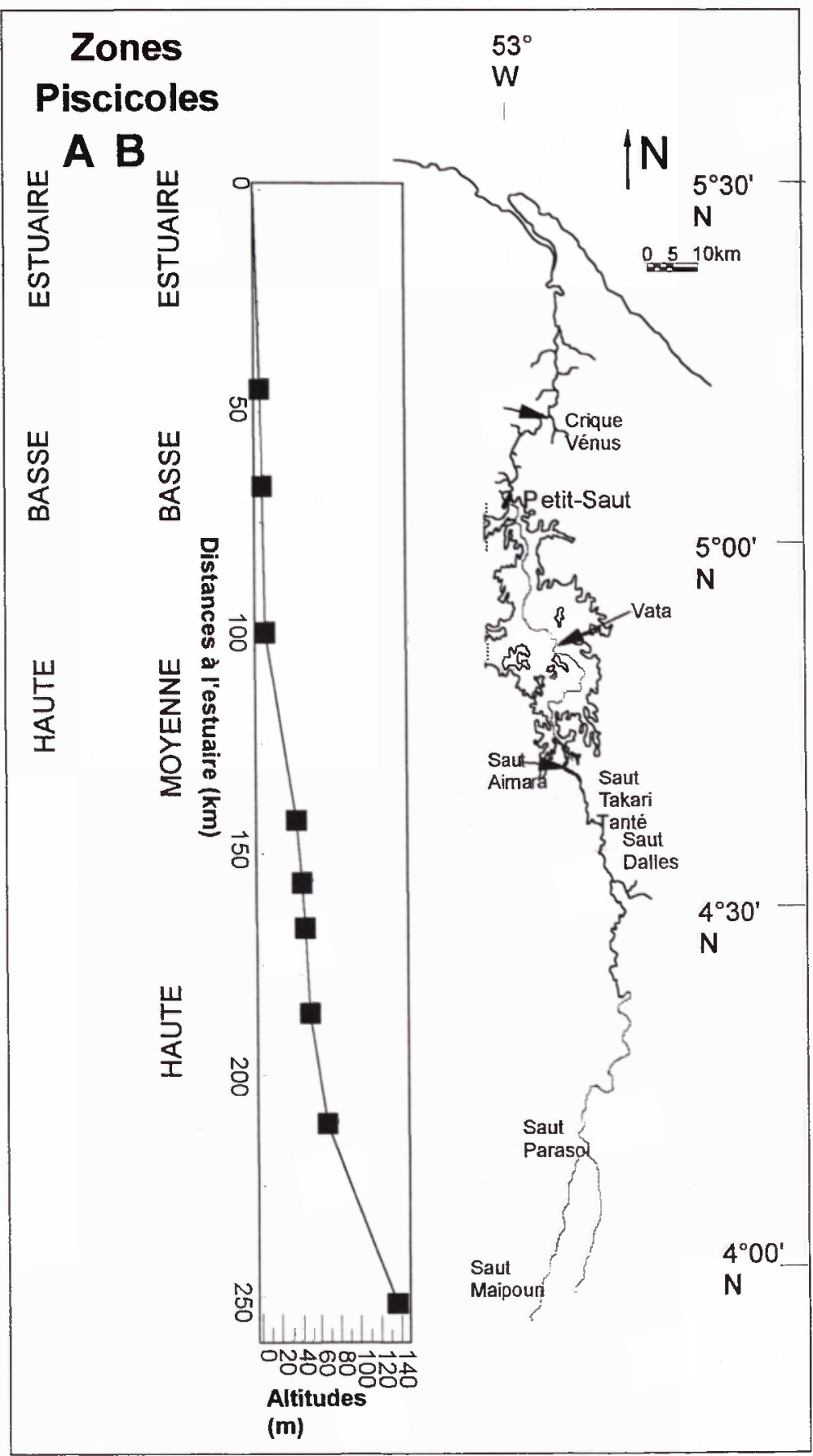


Depuis 1992 l'accent a été mis sur l'étude de la reproduction des poissons. Les époques de reproduction des espèces les plus abondantes ont été déterminées et des informations sur les tailles de première maturité obtenues. II apparaît que la plupart des espèces (22 sur les 24 examinées dans cette phase) ont une reproduction très étalée tout au long de la saison des pluies de décembre à juillet. Ces études, intensifiées dans la phase suivante, permettent de déterminer les différentes stratégies mises en place par les espèces de poissons dans ce milieu (Ponton \& Mérona, 1998). Celles-ci sont à la base de la capacité des espèces à se développer dans les milieux transformés par la fermeture du barrage.

Aucune étude de croissance n'a été effectuée. Seul l'examen des structures en taille pour les populations suffisamment échantillonnées permet d'estimer ce paramètre. La taille maximale observée est également un indicateur de la croissance.

\subsubsection{Etude des juvéniles}

Les phases larvaires et juvéniles constituent une étape fondamentale dans le cycle de vie des poissons. C'est en effet l'époque où les animaux sont les plus exposés aux facteurs de mortalité (maladies, prédation, dénutrition,...). Le succès de la reproduction, c'est à dire l'intensité du recrutement et donc le maintien des populations de poissons est donc étroitement liés aux événements vécus pendant cette phase. Cet aspect est depuis longtemps au centre des préoccupations des biologistes des pêches alors qu'il était très généralement ignoré dans les études d'impact particulièrement en milieu tropical. Pour combler cette lacune, un programme sur les juvéniles a donc été initié par l'ORSTOM en 1991. Dans la mesure où les études sur les juvéniles sont rares dans la zone géographique, la tâche était particulièrement difficile. II a fallu développer des méthodologies adaptées au milieu et au matériel biologique (Ponton, 1994). II était par ailleurs indispensable de décrire et déterminer les stades larvaires des espèces récoltées. Ainsi les premières données utilisables sur les jeunes poissons ont concerné la saison 1992-1993, qui a précédé la fermeture définitive du barrage en janvier 1994. La dynamique d'apparition des larves et juvéniles dans un milieu inondé du Sinnamary aval a donc pu être déterminée pendant une année avant la fermeture du barrage. Par ailleurs la structure des assemblages de juvéniles a été déterminée dans 10 affluents de la zone aval (Ponton \& Copp, 1997).

\subsubsection{Les prévisions d'impact}

La première phase de recherches a conduit à la formulation d'un certain nombre de prévisions sur le devenir des poissons après la formation du lac de retenue.

Les conséquences de la diminution de l'oxygène dissous tant dans la retenue qu'à l'aval de celle-ci ont été discutées à partir d'une analyse de la bibliographie (EDF, 1984; Planquette et al., 1985). Quelques espèces pré- 
sentes dans le Sinnamary sont connues pour leur capacité à utiliser l'air atmosphérique. Pour les autres, des seuils de tolérance sont proposés sur la base de quelques études ponctuelles.

Basées sur les principaux cas de grands barrages en zone intertropicale (Doudet, 1979; Kapetsky, 1978; Petr, 1967a et b; Richter \& Nijssen, 1980), un certain nombre de prévisions concernant la structure et la composition des peuplements ichtyques ont pu être avancées. Certaines sont d'ordre général et n'utilisent pas les connaissances acquises sur le Sinnamary telles qu'une probable augmentation de la taille maximale de certaines espèces, ou une diminution de la diversité tant dans le lac qu'à l'aval. D'autres s'efforcent de déterminer la possible composition et organisation des communautés de poissons mais, de l'avis même des auteurs, la connaissance des mécanismes qui régissent les réactions des peuplements de poissons aux facteurs de milieu sont insuffisantes pour avancer des prévisions précises.

Les travaux ultérieurs ont tenté d'aller plus loin dans l'anticipation des effets du barrage en testant un certain nombre d'hypothèses sur le fonctionnement du compartiment poisson dans le milieu. Ainsi par exemple le rôle des zones forestières inondées s'est avéré secondaire par rapport aux zones de bordure dans le cycle reproductif des espèces (Lauzanne et al., 1995). Ainsi aussi le rôle de l'estuaire dans la reproduction des espèces dulçaquicole de la portion aval, est, lui aussi apparemment négligeable (Tito de Morais et Tito de Morais, 1994).

\subsection{Les travaux après fermeture}

La mise en eau a duré environ 16 mois de janvier 1994 jusqu'en mai 1995. Pendant toute cette période, de multiples observations ont été pratiquées sur les peuplements de poissons dans les différents compartiments de la rivière barrée.

\subsubsection{Les effets de la mise en eau sur les peuplements de poissons adultes}

\subsubsection{Les variations d'abondance}

Comme cela a été souvent observé dans des situations de barrage, les captures ont été nettement plus élevées dans la retenue que dans la rivière pendant toute la phase de remplissage, ce qui suggère une densité importante de poissons (fig. 4). Deux hypothèses explicatives pourraient être qu'il y a eu pendant cette période : 1) une importante survie juvénile tout au long de la saison de reproduction du fait de l'inondation permanente de nouveaux milieux et peut-être d'une dispersion de leurs prédateurs, et 2) une survie adulte et un taux de croissance élevée du fait de l'abondance de sources de nourriture allochtone (végétation et invertébrés terrestres principalement).

2.2.1.2 La composition et les caractéristiques des peuplements

Pendant toute la phase de remplissage les peuplements de poissons ont 


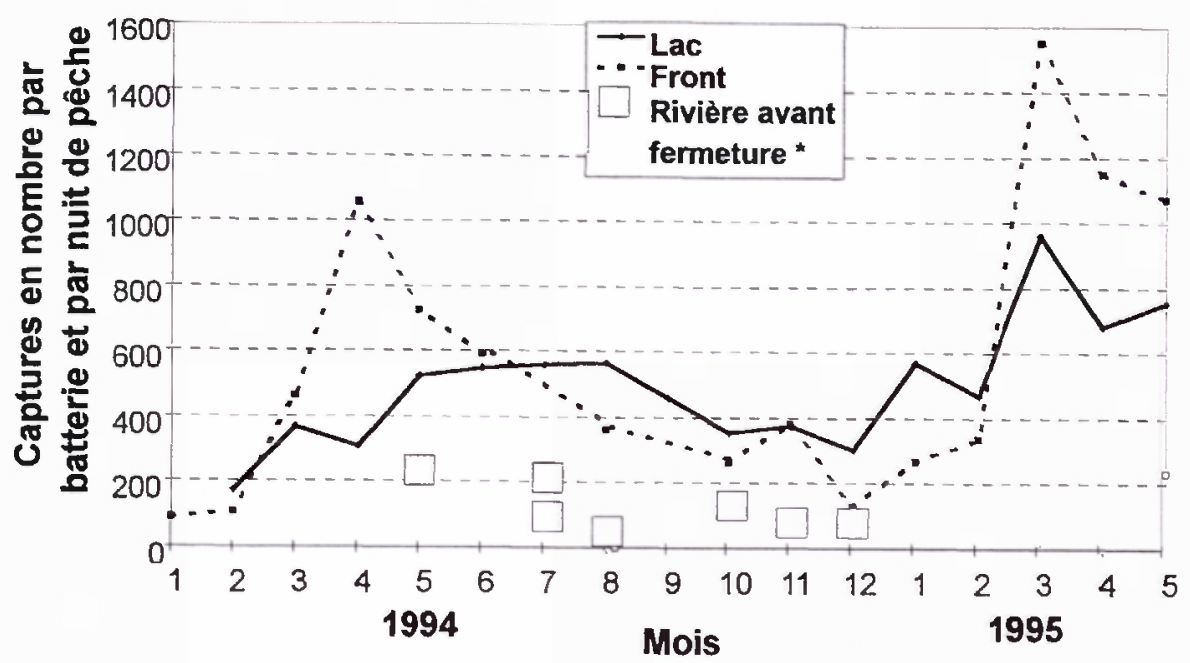

Fig. 4. - Variations des captures de poissons dans la retenue de Petit-Saut pendant la phase de remplissage et comparaison avec les données de captures effectuées avant barrage.

* Captures dans les stations L'Autel, Aimara et Takari Tanté actuellement noyées.

Fig. 4. - Fish catch variations in Petit-Saut reservoir during the filling stage. Comparison with the preimpoundment catches.

*Catches in the stations: L'Autel, Aimara and Takari Tante, now flooded.

montré une relative stabilité. Les mêmes 7 espèces dominent le peuplement, ce sont Parauchenipterus galeatus, Triportheus rotundatus, Charax gibbosus, Cyphocharax sp., Acestrorhynchus falcatus, Bryconops $s p$. et Leporinus friderici. II s'agit vraisemblablement de celles qui ont profité les premières des conditions favorables à leur reproduction. Par ailleurs, dans la partie centrale de la retenue qui est la seule échantillonnée, le peuplement ichtyque, est relativement homogène. Enfin, dans cette première phase il n'y a pas de diminution apparente de la richesse spécifique. De nombreuses espèces benthiques parviennent à se maintenir dans la couche superficielle oxygénée (par ex. Hypostomus plecostomus).
2.2.1.3 Les caractéristiques des espèces

Pendant toute la phase de mise en eau, les informations sur les stratégies de reproduction ont été complétées. En particulier la fécondité de la plupart des espèces a été déterminée et les tailles de première maturité déterminées (Ponton \& Mérona, 1998).

Les 7 espèces les plus abondantes dans la retenue présentent deux types de stratégie. L'une d'entre elles (Parauchenipterus galeatus) pratique une reproduction continue tout au long de l'année. Son régime alimentaire est de type omnivore opportuniste. Cette stratégie de généraliste lui confère vraisemblablement un avantage compétitif lors de perturbations 
qui libèrent brusquement de grandes quantités de ressources tant spatiales qu'alimentaires, jusque là limitées dans le milieu. Les autres espèces ont une reproduction saisonnière avec une ponte parcellée. Elles présentent des régimes alimentaires variés. Une hypothèse pour expliquer leur succès immédiat pourrait être une adéquation temporelle entre leurs pontes et la montée continue des eaux du lac qui aurait entraîné un succès important de leur reproduction pendant la crue de 1994.

\subsubsection{Les effets de la fermeture du barrage sur les peuplements adultes à l'aval}

\subsubsection{Les variations d'abondance}

La partie aval du fleuve était suivie dans une seule station située à égale distance entre le barrage et l'estuaire. Là, on a assisté à une diminution importante des captures toutes espèces confondues. Cette observation a été cependant difficile à interpréter. D'une part la baisse observée pouvait être due partiellement à une diminution de la capturabilité du fait d'une dégradation de la qualité physique de l'eau. L'eau du Sinnamary s'est chargée de matériel en suspension provenant de la dégradation d'algues filamenteuses et ce matériel provoquait un colmatage des filets. D'autre part, une diminution de l'abondance de poissons dans cette portion de la rivière ne disait rien sur leur présence ou absence dans l'ensemble de la zone aval et en particulier sur l'existence de zones refuges. Certaines mortalités ponc- tuelles de poissons ont été observées plus en aval mais ni le nombre, ni la biomasse, ni même la composition spécifique de ces poissons morts n'ont pu être déterminés.

\subsubsection{Caractéristiques et composi- tion des peuplements}

Parallèlement à la baisse dans les captures, on observe une diminution de la richesse spécifique dans les échantillons. La richesse cumulée passe de 54 espèces dans les 13 échantillons avant barrage à 46 dans les 14 échantillons réalisés entre janvier 1994 et mai 1995 La régularité des échantillons augmente immédiatement après la fermeture du fait de leur extrême pauvreté. Ainsi, en mars 1994, 6 poissons appartenant à 6 espèces différentes ont été capturés. Avec la reprise de captures plus significatives, la régularité diminue et une seule espèce devient très dominante (Curimata cyprinoides). Cette espèce qui présente une reproduction saisonnière et une alimentation basée sur les algues semble ainsi avoir bien résisté aux événements liés à la fermeture du barrage.

\subsubsection{Les effets de la fermeture du barrage sur les juvéniles à l'aval}

2.2.3.1 Effets sur l'occurrence, la diversité et la densité des juvéniles

Les jeunes poissons ont été suivis pendant toute la durée de la saison des pluies correspondant à la phase de remplissage du lac de retenue avec la même méthodologie em- 
ployée l'année précédente (Ponton, 1995; Ponton et Vauchel, à paraître). Les résultats font apparaître trois types de modifications:

- La séquence d'apparition des jeunes a été fortement modifiée par rapport à la situation avant barrage.

- Le nombre de taxons correspondant à des jeunes stades a fortement diminué. Sur les 37 taxons observés en 1992-93, seuls 29 sont présents dans les échantillons de la saison 93-94.

- La quantité de ces individus jeunes a elle aussi fortement diminué (fig. 5). La densité de jeunes est marquée par une augmentation importante en fin de saison des pluies, à partir du mois de mai en 1992-93. Les captures par effort peuvent atteindre à ce moment plus de 60 individus par piège et par nuit. En 1993-94 ce pic de densité n'apparaît pas et les captures restent de l'ordre de quelques individus par piège et par nuit.

Ces modifications sont liées à la modification du régime hydrologique imposée par les opérations de remplissage de la retenue (Ponton et Vauchel, à paraître). La crue "normale" du fleuve a en effet été presque totalement supprimée et, hormis quelques lâchés d'eau correspondant à des paliers techniques, le niveau du fleuve est resté bas (fig. 6). La conséquence en a été une diminution drastique de l'étendue des zones inondées au niveau des embouchures des criques et une modification de l'hydrodynamique criques et fleuve.
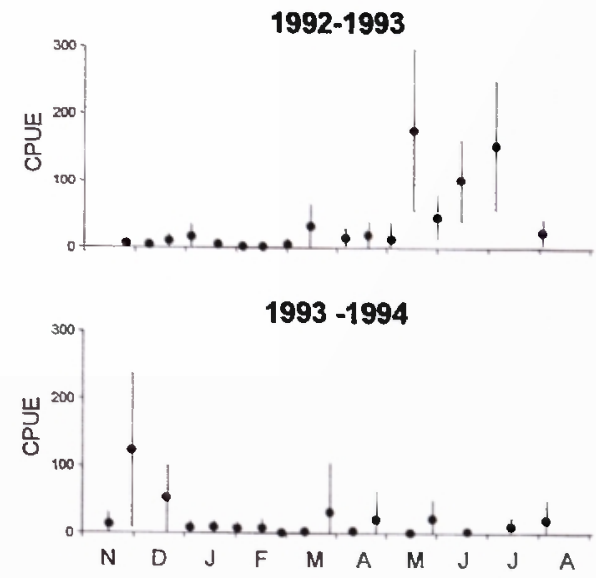

Fig. 5. - Variations des captures par effort de juvéniles dans les nasses lumineuses pendant les saisons $1992 / 93$ et $1993 / 94$ dans la crique Vénus (affluent rive droite de Sinnamary à l'aval du barrage de Petit-Saut). D'après Ponton (1995).

Fig. 5. - Variations of the catch by effort for the juveniles in light traps, during the seasons 1992/93 and 1993/94, in crique Venus (right bank tributary of the Sinnamary river, downstream from the Petit-Saut dam). From Ponton (1995).

2.2.3.2 Effets sur la composition des assemblages

L'examen de la répartition des différents ordres de poissons représentés dans les échantillons récoltés dans une dizaine de criques à l'aval en 1993, puis en 1994 dans les différentes zones du système (Aval, Lac de retenue, Tète du lac, et amont) fait apparaître une modification radicale des assemblages de l'aval pendant l'année de remplissage (Ponton et Copp, 1997). En 1993, les assemblages étaient dominés par les Characiformes, les Perciformes ne représentant que $13 \%$ des individus. 


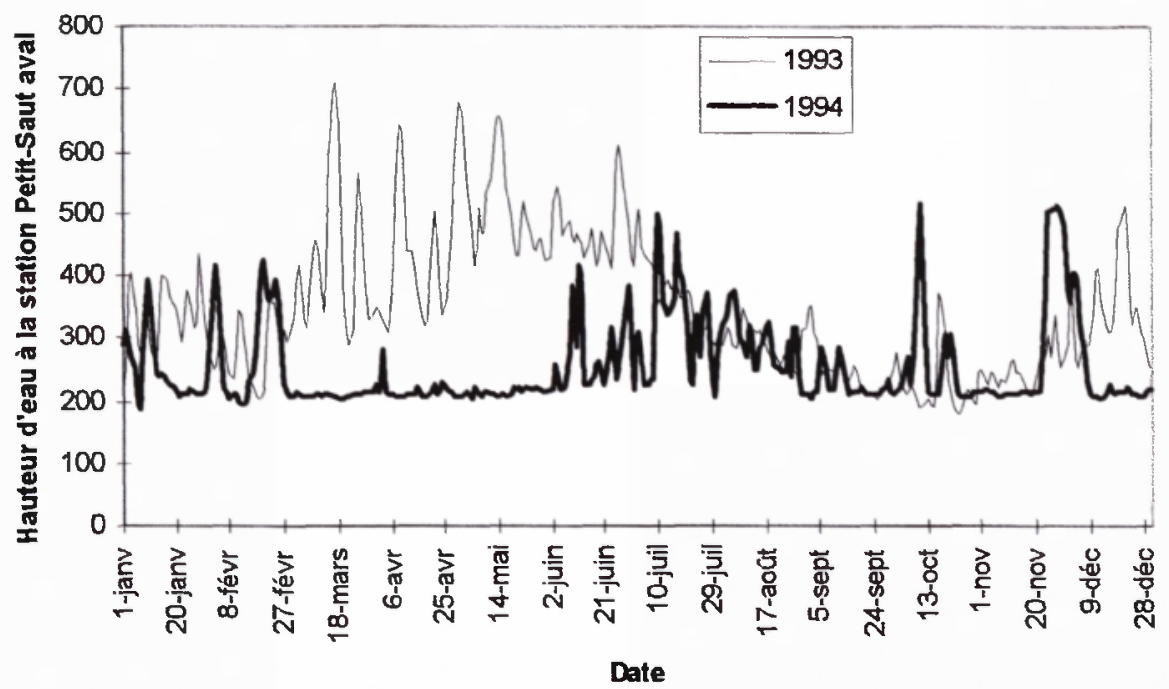

Fig. 6. - Variations des hauteurs d'eau journalières à la station limnimétrique de Petit-Saut Aval en 1993 et 1994.

Fig. 6. - Daily variations of the water level at the limnimetric station Petit-Saut Aval in the years 1993 and 1994.

En 1994, dans les mêmes stations, les Perciformes deviennent majoritaires avec $55 \%$ des individus. Cette observation est à rapprocher des études sur les stratégies de vie des poissons. Les Characiformes sont en effet le plus souvent des poissons à la reproduction saisonnière qui produisent beaucoup d'œufs et ne présentent pas de comportement parental de protection de ces œufs. Ainsi la survie des larves est étroitement dépendante de la crue qui les transporte passivement dans des milieux favorables à leur survie. A l'inverse beaucoup de Perciformes ont des comportements de protection de leur ceufs et de leur jeunes qui permettent d'optimiser leur survie quelque soient les conditions pourvu qu'elles ne soient pas létales.

\subsubsection{Les études dans la phase d'évolution écologique}

\subsubsection{Adultes dans la retenue}

Le suivi rapproché des populations de poissons adultes s'est poursuivi dans la retenue sur un rythme de tournées d'échantillonnage mensuelles jusqu'en décembre 1996 (Mérona, 1997b). Pendant toute cette période les captures se sont maintenues à un niveau élevé tant dans la retenue ellemême qu'au niveau de son contact amont avec la rivière (le front). Un cycle saisonnier semble se mettre en place avec un maximum en début de crue (janvier-février) et un minimum en fin d'étiage (novembre-décembre). En outre, une hétérogénéité spatiale apparaît, les captures étant moins éle- 
vées dans la partie basse de la retenue et dans les milieux fermés que dans la partie moyenne et dans les eaux libres. La richesse spécifique dans la retenue subit une baisse régulière depuis la fermeture alors que la régularité, dont les valeurs sont fajbles par rapport à celles observées dans la rivière, ne varie pas sur toute la période. En ce qui conceme la composition spécifique, les assemblages dans la retenue se distinguent nettement de ceux du front qui eux sont proches de ceux de la rivière. Ils sont homogènes dans toute la partie axiale du lac mais on a assisté, depuis la fermeture à des remplacements d'espèces parmi les plus abondantes.

L'analyse de ces résultats a conduit à la définition d'un suivi sur le long terme basé sur deux observations annuelles, l'une en fin de crue (juin-juillet) et l'autre en fin de décrue (novembre).

\subsubsection{Relations retenue-amont}

Une opération visant à étudier les échanges entre la retenue et la portion amont du fleuve a été lancée en octobre 1995. Les objectifs sont d'une part de vérifier les hypothèses d'un modèle source/puits qui explique la persistance globale d'une espèce dans une aire donnée par la migration d'individus de zones démographiquement excédentaires (le lac) vers les zones démographiquement déficitaires (le tronçon amont). II sera possible d'autre part d'évaluer l'ensemencement du lac par des espèces se reproduisant à l'amont.
2.2.4.3 Les poissons adultes à l'aval

Les études à l'aval sur les poissons adultes se sont fixé pour but de compléter les informations recueillies par une vision plus globale de la zone. Des échantillons ont été pratiqués dans 4 stations, réparties sur l'ensemble du tronçon aval hors coin salé. Les résultats font apparaître une importante accumulation de poissons au pied de la digue, au niveau des évacuateurs de fond. Ce phénomène a été attribué d'une part aux bonnes conditions d'oxygénation de la zone et d'autre part à des mouvements migratoires bloqués par la présence du barrage (Mérona et al., 1997). Après la fin de la phase de mise en eau et le retour à un cycle de crue normal, les captures reviennent à un niveau comparable à ce qu'il était avant barrage (Albert, 1996; Mérona et al., 1997). En revanche, la richesse spécifique et la régularité diminuent encore. Certaines familles sont favorisées par les nouvelles conditions telles les Curimatidae, les Sternopygidae et les Cichlidae, d'autres présentent la tendance inverse telles les Engraulidae et les Characidae. Ces évolutions ne semblent pas liées à la stratégie de reproduction de ces espèces, mais bien plutôt à leur mode d'alimentation.

\subsubsection{Les relations entre l'habitat et les communautés de juvéniles}

Les études sur les juvéniles se sont focalisées sur les facteurs de l'habitat aquatique structurant les communautés de juvéniles à différentes échelles d'espace et de temps. S'appuyant sur 
les traits biologiques des espèces présentes, ces études se proposent d'examiner deux hypothèses principales :

- les conditions environnementales annuelles particulières, notamment hydrologiques, dans les trois secteurs considérés (amont de la retenue, aval de la retenue et retenue) structurent fortement les communautés de juvéniles de poissons dans ces milieux,

- les variations saisonnières du régime hydrologique dues à l'alternance d'une saison sèche et d'une saison humide ainsi que l'hétérogénéité du milieu influent sur la distribution des juvéniles dans les différents affluents étudiés (compartiments).

Les résultats escomptés permettront une généralisation des effets de la perturbation induite par un barrage par l'utilisation de descripteurs fonctionnels affranchis du cadre taxonomique propre à la Guyane.

\section{LE RETOUR D'EXPÉRIENCE}

Compte tenu du schéma général proposé par le SCOPE (1972) et du bilan des études sur les poissons réalisées à Petit-Saut, il est possible de lister les points positifs et les difficultés rencontrées.

\subsection{La chronologie}

II est clair que des considérations sur les poissons, de même d'ailleurs que sur d'autres paramètres environ- nementaux, n'ont pas été pris en compte dans le processus de décision. Considérant le problème a posteriori, il semble bien que des études ichtyologiques préliminaires auraient été favorables au choix du Sinnamary. II y a d'une part comme argument l'absence d'espèce endémique. II y a d'autre part les caractéristiques du régime hydrologique très variable de ce fleuve. Les populations de poissons ont dû s'adapter à cette variabilité imprévisible. Or on constate que beaucoup de ces populations présentent des stratégies de reproduction basées sur des pontes multiples alors que les mêmes espèces en milieu à la variabilité plus prévisible ont des pontes uniques. Ainsi cette stratégie qui, comme le suggère Lowe-McConnell (1987), pourrait être une adaptation aux fluctuations imprévisibles de la hauteur d'eau, donnerait à ces espèces une plus grande capacité à surmonter la perturbation due au barrage. II reste bien sûr à vérifier dans quelle mesure elles pourront effectivement, sur le long terme, surmonter l'épisode de la mise en eau qui représentait une perturbation sans commune mesure avec celles observées naturellement.

\subsection{L'utilisation des expériences passées}

Le barrage de Petit-Saut est loin d'avoir été la première situation tropicale où des études environnementales ont été menées. Dès les années 50 , la création du lac Kariba a été l'occasion de nombreuses études synthétisées dans un ouvrage (Balon et 
Coche, 1974). Les lacs Nasser, Volta, Kainji, Kossou, en Afrique, Brokopondo, Tucurui, Itaipu, Guri, en Amérique du Sud ont également été suivis avec plus ou moins d'intensité (LoweMcConnell, 1966; Obeng, 1969; Ackermann et al., 1973; Bernacsek, 1984; Straskraba et al., 1993). La prise en compte de ces expériences passées se heurte à un certain nombre de difficultés. D'une part les données disponibles sont hétérogènes: ici quelques échantillonnages ponctuels, là un suivi d'une pêcherie commerciale, des méthodologies diverses. D'autre part, le cadre taxonomique est différent d'une situation à une autre et il est difficile de comparer les assemblages et les espèces une à une. L'expérience de Petit-Saut montre qu'une analyse en terme de stratégies est possible et permettrait une réelle comparaison de systèmes éloignés géographiquement. Par ailleurs un grand nombre de données ne sont pas disponibles, restant sous forme de rapports d'obtention très difficile. Le résultat est donc le plus souvent une utilisation très anecdotique des expériences passées qui ne permet pas de prévisions très précises à partir de la connaissance approfondie de la situation étudiée.

\subsection{Les problèmes taxonomiques et l'intervention de la génétique}

Le travail d'identification de la faune, préalable indispensable à toute étude d'écologie est toujours très long et ses résultats pas toujours satisfaisants. Depuis plusieurs années l'utilisation de la génétique pour identifier les populations animales et en particulier les poissons, s'est beaucoup développée. Un travail a même été effectué en Guyane et a donné des résultats encourageants (Renno et al., 1989). L'intervention de spécialistes de la discipline aux tous débuts du programme auraient vraisemblablement permis d'aller plus vite dans cette tâche et de résoudre plus de problèmes.

\subsection{Les problèmes de l'oxygène}

L'occurrence de mortalités de poissons pendant la phase de remplissage à l'aval du barrage a fait surgir de nombreuses questions parmi la population humaine. L'oxygène était-il réellement en cause? Quelles pouvaient être les causes alternatives? Quelle était l'importance relative de ces mortalités? II faut admettre que les ichtyologistes étaient fort embarrassés pour répondre à ces questions. II y a à cela plusieurs raisons. D'une part le phénomène limité dans le temps à quelques épisodes n'a pu être étudié en détail. Ni la composition spécifique des mortalités, ni leur importance pondérale, ni les conditions limnochimiques régnant à l'endroit précis du problème n'étaient disponibles.

Par ailleurs, la détermination des seuils de tolérance des espèces du Sinnamary à la déficience à l'oxygène et à la concentration en produits toxiques est un travail considérable et complexe qui dépasse probablement le cadre d'études d'environnement associées à un projet de développe- 
ment. La tolérance des poissons aux faibles teneurs d'oxygène dissous est en effet un problème complexe avec des aspects, anatomiques, comportementaux, physiologiques et biochimiques (Val et Almeida-Val, 1995).

Face à ces difficultés, la stratégie la plus efficace dans l'optique d'un retour d'expérience semble être la mise en place d'une surveillance rapprochée qui permettrait la mobilisation rapide d'équipes lorsqu'il y a risque de déficits d'oxygène.

\subsection{L'approche stratégie vitale : seul moyen de généraliser les ob- servations}

L'analyse des premiers résultats des études sur les peuplements de poissons dans le Sinnamary barré conforte le bien fondé de l'accent mis sur l'approche stratégie vitale. Cette approche qui a bénéficié de travaux récents en écologie théorique (Winnemiller, 1989, 1992; Bruton, 1990) présente deux avantages fondamentaux :

- elle permet d'émettre des hypothèses sur les raisons du maintien ou du développement de certaines espèces au détriment d'autres,

- elle permet la généralisation des observations à d'autres cas de barrages dans des contextes taxinomiques différents. Ainsi il devrait être possible de réexaminer des données historiques sous cet angle et de tenter de dégager des patrons de réaction des peuplements aux nouvelles conditions induites par un barrage.

\subsection{La problématique jeunes poissons}

Cette opération de recherches qui constituait une première dans le cadre d'étude d'impact de barrages en zone tropicale s'est avérée riche en enseignements. Elle a permis le développement d'une approche holistique dans l'étude des peuplements de poissons. En effet l'ensemble des phénomènes intervenant entre la ponte et l'adulte est souvent considéré comme une "boîte noire" alors qu'il s'agit d'une phase critique de la vie du poisson pendant laquelle vont pouvoir être détectés rapidement les effets de perturbations sur les populations. Dans le Sinnamary aval le suivi des assemblages de juvéniles a seul permis de détecter de manière claire les effets de la fermeture et de formuler des hypothèses sur les mécanismes d'impact.

Par ailleurs, les méthodologies d'échantillonnage développées pour la capture des jeunes poissons permettent aussi la capture des petites espèces qui échappent aux moyens traditionnels de captures tels que les filets. Or ces espèces, régulièrement négligées, s'avèrent majoritaires dans la plupart des peuplements de poissons néotropicaux.

\subsection{L'importance de l'hydrologie}

Le déroulement des travaux sur les jeunes poissons et les petites espèces a fait apparaître l'importance fondamentale des processus hydrologiques à une échelle fine. L'interpré- 
tation des résultats obtenus auraient pu être beaucoup plus poussée si on avait disposé d'études préliminaires ou au moins parallèles sur l'hydrologie à différentes échelles d'espace. Ainsi la modélisation de la propagation de l'onde de marée à l'aval est intervenue tardivement, après la fermeture. Ainsi également l'établissement de relations entre la surface des zones inondées et les hauteurs d'eau du fleuve qui auraient permis d'interpréter les variations de densité de juvéniles. Enfin les résultats suggèrent que les relations fonctionnelles entre les criques et le fleuve ont probablement une influence prépondérante sur le comportement tant des jeunes poissons que des adultes des espèces de taille moyenne ou grande, qui, à certaines époques utilisent les criques. Une analyse fine de ces relations aurait donc été nécessaire.

\section{CONCLUSIONS ET RECOMMANDATIONS}

A l'issue de ce bilan rapide centré sur les études ichtyologiques à PetitSaut, il est possible de suggérer un certain nombre de recommandations pour optimiser l'investissement toujours important dans les études environnementales pour la construction de futurs barrages.

La première d'entre elles est liée aux difficultés rencontrées dans la prise en compte des expériences passées. Des prévisions pertinentes ont pu être réalisées en utilisant des don- nées historiques (Marshall, 1984; Bernacsek, 1984). Cependant, le travail qui permettrait une utilisation plus efficace de ces données historiques demande un investissement important et ne peut être réalisé au sein d'un programme d'impact sans moyen humain et financier spécialement dédiés à cette tâche. II y a donc là, à notre sens une réelle nécessité d'une opération de recherche particulière, non nécessairement liée à la construction d'un ouvrage particulier. II est possible de déterminer pratiquement comment ce programme pourrait s'articuler. Dans un premier temps un réseau de correspondants localisés dans chacun des pays où de grands barrages ont été réalisés devrait être constitué. Ces correspondants auraient pour tâche de collecter et synthétiser les données produites sur l'environnement dans les projets de barrage. Une base de données serait ainsi constituée en homogénéisant au maximum les différentes informations disponibles. Des traitements seraient ainsi possibles pour tenter de mettre en relation les différentes caractéristiques des ouvrages à nombre de paramètres physico-chimiques et biologiques.

Un deuxième groupe de recommandations concerne la chronologie des travaux d'environnement.

Le choix de l'énergie hydroélectrique d'abord, puis celui du site, repose généralement sur des considérations sociales et économiques (Church, 1968).

La prise en compte, souhaitable, des données environnementales dans la décision de construction d'un bar- 
rage hydroélectrique implique, comme nous l'avons vu, des études comparatives antérieures à la prise de décision. Ces études doivent s'appuyer sur l'analyse des retours d'expériences des nombreux ouvrages réalisés dans le passé (voir paragraphe précédent) et sur des observations à grande échelle de la situation envisagée.

Passée cette phase, lorsque les travaux sont sur le point de commencer, il est essentiel de mener une réflexion approfondie en vue de construire un programme d'études intégrées, seul garant de la fiabilité des prévisions d'effet. Cette réflexion, nécessairement multidisciplinaire, est le rôle d'un comité d'experts indépendants. La mise en route du programme ainsi proposé est évidemment longue. II faut mobiliser les équipes et les moyens. C'est pourquoi cette étape incontournable doit intervenir très tôt dans le processus.

II est probable et souhaitable que cette réflexion, menée par des spécialistes de différentes disciplines, conduise à proposer des études sur le fonctionnement et sur les mécanismes focalisées sur des questions précises, dans lesquelles l'ichtyologie serait un aspect intégré dans une problématique plus vaste. Ce type d'approche pourrait nous conduire à diriger les études sur les poissons de manière sensiblement différente de celle qui a été adoptée dans le cas de Petit-Saut. Néanmoins, quelque soit la problématique l'expérience de Petit-Saut conduit à recommander pour les études ichtyologiques :
- l'intervention de la génétique dans les inventaires faunistiques,

- une approche holistique de l'étude des peuplements de poissons incluant toutes les phases du cycle de vie des espèces, depuis la phase larvaire jusqu'à la phase adulte,

- une approche basée sur les stratégies de vie des populations et leur relation avec l'environnement, seule à même de dépasser le cadre régional de l'ouvrage.

Enfin la prolongation des études sur le long terme apparaît absolument indispensable pour une évaluation des effets réels du barrage. Ce type de travaux est rarement réalisé du fait d'un certain nombre de difficultés telles que la garantie de financements ou la mobilisation d'équipes sur de longues périodes. Pourtant un suivi de ce type ne demanderait qu'un financement réduit et la situation de PetitSaut avec une infrastructure existante, dans un environnement socio-politique stable constitue une opportunité rare de pouvoir réaliser une telle opération.

\section{RÉFÉRENCES BIBLIOGRAPHIQUES}

Ackermann W.C., White G.F. \& Worthington E.B. (eds.), 1973. Man-made lakes: their problems and environmental effects. American Geophysical Union. Washington D.C. : 847 p.

Albert P., 1996. Suivi écologique des communautés de poissons en aval du barrage de Petit-Saut (Guyane fran- 
çaise). Rapport de stage. ORSTOM Cayenne: $74 \mathrm{p}$.

Balon E.K. \& Coche A.G., 1974. Lake Kariba: A man-made tropical ecosystem in Central Africa. Dr. W. Junk Pub., The Hague : $767 p$.

Bernacsek G.M., 1984. Guidelines for dam design and operation to optimize fish production in impounded river basins (based on a review of the ecological effects of large dams in Africa). CIFA Tech. Pap. (11) : 98 p.

Boujard T. \& Rojas-Beltran R., 1988. Zonation longitudinale du peuplement ichtyque du fleuve Sinnamary (Guyane française). Rev. Hydrobiol, trop. 21(1) : 47-61.

Bruton M.N., 1990. Trends in the life-history styles of vertebrates : an introduction to the second ALHS volume. Environ. Biol. Fishes $28: 7-16$.

Church R.J.H., 1968. A geographical view. In Dams in Africa, W.M. Warren \& N. Rubin eds. Frank Cass \& Co. Ltd. Pub. : 1-12.

Doudet T., 1979. La pêche artisanale dans la retenue artificielle d'Ayamé (Côte d'Ivoire). C.T.F.T. Notes et documents sur la pêche et la pisciculture 19:126.

EDF, 1984. Aménagement du Sinnamary. Chute de Petit-Saut. Demande de concession et demande de déclaration d'utilité publique. : 149-189.

Horeau V., Cerdan P., Champeau A. \& Richard S., 1996. Importance des aliments d'origine allochtone pour les poissons dans des criques du bassin du Sinnamary (Guyane française). Revue d'Ecologie - La Terre et la Vie $51(1): 29-41$.

Kapetsky J.M., 1978. The Brokopondo reservoir fishery yield, fishery research and fishery development. F.A.O., Rome: $66 \mathrm{p}$.

Karr J.R., Fausch K.D., Angermeier P.L., Yant P.R., Schlosser I.J., 1986. Assessing biological integrity in running waters. A method and its rationale. Illinois
Natural History Survey, Special Publication $5: 28 \mathrm{p}$.

Lauzanne L., Tito de Morais L., Ponton D., Mérona B. de, Bron J.-C., Raffray J., Tarcy M., Mallet A., Brehm N. \& Besançon A., 1995. Structure et biologie des peuplements ichtyques du fleuve Sinnamary en Guyane française. Rapport final, Convention EDF N $N^{\circ} \mathrm{GP}$ 7530. ORSTOM Cayenne: $139 \mathrm{p}$.

Lauzanne L., Tito de Morais L., Tito de Morais A. \& Ponton D., 1993. Structure et biologie des peuplements ichtyques du fleuve Sinnamary en Guyane française. Rapport final, Contrat EDF $N^{\circ}$ GP 7514. ORSTOM Cayenne: 133 p. + annexes.

Lowe-McConnell R.H.,(ed.) 1966. Manmade lakes. Proceedings of a Symposium held at the Royal Geographical Society, London. 30 sep. $1^{\text {er }}$ oct. 1965. Academic Press. London : $218 \mathrm{p}$.

Lowe-McConnell R.H., 1987. Ecological studies in tropical fish communities. Cambridge University Press. Cambridge : $382 \mathrm{p}$.

Marshall B.E., 1984. Towards predicting ecology and fish yields in African reservoirs from preimpoundment physico-chemical data. Comment prévoir l'écologie des réservoirs africains et leur rendement en poisson à partir de données physico-chimiques réunies avant endiguement. CIFA Tech. Pap./Doc. Tech. CPCA, $12: 36 \mathrm{p}$.

Mérona B. de, 1985. Les peuplements de poissons et la pêche dans le bas Tocantins (Amazonie brésilienne) avant la fermeture du barrage de Tucurui. Verh. Internat. Verein. Limnol. 22 : 2698-2703.

Mérona B.de (ed.), 1997a. Structure et biologie des peuplements ichtyques du fleuve Sinnamary en Guyane française. Rapport terminal, Convention EDF N N GP 7572. ORSTOM Cayenne: $157 \mathrm{p}$.

Mérona B. de, 1997b. Suivi écologique des communautés de poissons dans 
le Réservoir de Petit-Saut. In Rapport terminal, Convention EDF $N^{\circ}$ GP 7572. ORSTOM Cayenne: 34-62.

Mérona B.de, Albert P., Tito de Morais L., Lauzanne L., 1997. Suivi écologique des communautés de poissons adultes à l'aval du barrage de PetitSaut. In Rapport terminal, Convention EDF N N $N^{\circ} \mathrm{7572}$. ORSTOM Cayenne: $5-33$.

Mérona B. de, Ponton D., Guégan J.-F., Mérigoux S., Bron J.-C., Tarcy M., Ruffine R. \& Brehm N., 1996. Structure et biologie des peuplements ichtyques du fleuve Sinnamary en Guyane francaise. Rapport intermédiaire, Convention EDF $N^{\circ}$ GP 7572. ORSTOM Cayenne: $45 \mathrm{p}$.

Obeng L.,(ed.) 1969. Man-made lakes. The Accra Symposium. Ghana Universities Press. Accra : 398 p.

Petr T., 1967a. Fish population changes in the Volta lake in Ghana during the first sixteen months. Hydrobiologia (2) : 30-42.

Petr T., 1967b. On some factors associated with the initial high fish catches in new African man-made lakes. Arch. Hydrobiol. 75 : 32-49.

Planquette P., Rojas-Beltran R. \& Le Bail P.Y., 1985. Etude de l'impact du projet d'aménagement hydroélectrique de Petit-Saut (Fleuve Sinnamary, Guyane) sur le peuplement ichtyologique. Rapport définitif. INRA, Kourou: $158 \mathrm{p}$.

Ponton D., 1994. Sampling neotropical young and small fishes in their microhabitats : an improvement of the quatrefoil light-trap. Arch. Hydrobiol. 131 (4) : 495-502.

Ponton D., 1995. Juvéniles. In Lauzanne et al. (eds.) "Structure et biologie des peuplements ichtyques du fleuve Sinnamary en Guyane française. Rapport final, Convention EDF $N^{\circ}$ GP 7530. ORSTOM Cayenne $»$ : 15-42

Ponton D. \& Copp G., 1997. Early dryseason assemblage structure and ha- bitat use of young fish in tributaries of the River Sinnamary (French Guiana, South America) before and after hydrodam operations. Environ. Biol. Fish., sous presse.

Ponton D. \& Mérona B.de, 1998. Fish lifehistory tactics in a neotropical river with a highly stochastic hydrological regime: the Sinnamary River, French Guiana, South America. Pol. Arch. Hydrobiol., 45(2), sous presse.

Ponton D. \& Vauchel P.. Immediate downstream effects of the Petit-Saut dam on young neotropical fish in a large tributary of the Sinnamary River (French Guiana, South America). Soumis à Regulated Rivers: Research and Management.

Renno J.-F., Guyomard R., Boujard T. \& Bastide C., 1989. Evidence for genetic isolation among four morphological species of Leporinus (Anostomidae, Pisces) in French Guiana. Aquatic Living Ressources, 2 ; 127-134.

Richter C.J.J. \& Nijssen H., 1980. Notes on the fishery potential and fish fauna of the Brokopondo reservoir (Surinam). Fish. Mgmt. 11(3) : 119-130.

SCOPE, 1972. Man-made lakes as modified ecosystems. ICSU-SCOPE, Paris : $76 \mathrm{p}$.

Straskraba M., Tundisi J.G. \& Duncan A., 1993. Comparative reservoir limnology and water quality management. Kluwer Academic Publishers. Dordrecht : $291 \mathrm{p}$.

Tito de Morais L. \& Planquette P., 1991. Peuplements ichtyologiques du Haut Sinnamary, Guyane française. Rapport final de Convention. ORSTOM Cayenne et INRA Guyane : 49 p. + annexes.

Tito de Morais L. \& Tito de Morais A., 1994. The abundance of larval and juvenile fish in a tropical estuary. Estuaries, 17(1B) : 227-236.

Val A.L. \& Almeida-Val V.M.F., 1995. Fishes of the Amazon and their Environment. Physiological and Biochemi- 
cal aspects. Springer-Verlag Berlin: Winnemiller K.O., 1989. Patterns of vari$224 \mathrm{p}$.

Verneaux J., 1981. Les poissons et la qualité des cours d'eau. Annales Scientifiques de l'Université de Franche-Comté Besançon, $4^{\mathrm{ème}}$ ser., fasc. 2 : 33-41. tion in life history among South American fishes in seasonal environments. Oecologia 81 : 225-241.

Winnemiller K.O., 1992. Life-history strategies and the effectiveness of sexual selection. OIKOS 63(2) : 318-327

LOUIS - JEAN

avenue d'Embrun, 05003 GAP cedex

Tél. : 04.92.53.17.00

Dépôt légal : 367 - Avril 1998

Imprimé en France 